\title{
Optimal joint transmission and harvested energy scheduling for renewable energy harvesting enabled cellular network under coordinated multi-point transmission
}

\author{
Zejue Wang ${ }^{1}$, Hongjia $\mathrm{Li}^{1}{ }^{*}$, Dan $\mathrm{Hu}^{2}$ and Song $\mathrm{Ci}^{3}$
}

\begin{abstract}
On-grid energy consumption of base stations (BSs) contributes up a significant fraction of the total carbon dioxide $\left(\mathrm{CO}_{2}\right)$ emissions of cellular networks, among which remote radio units (RRUs) absorb most of the energy consumption. To eliminate the on-grid energy consumption and the corresponding $\mathrm{CO}_{2}$ emission, we propose a new transmission framework, in which all RRUs and associated power amplifiers (PAs) are powered by hybrid energy sources including on-grid energy source and off-grid renewable energy source. Based on the framework, we pursue a systematic study on the joint transmission and harvested energy scheduling algorithm for the hybrid energy powered cellular transmission system under coordinated multi-point (CoMP) transmission. Firstly, we formulate an optimal offline transmission scheduling problem with a priori knowledge about channel state information (CSI), under constraint of available amount of harvested energy and stored energy at each transmission time interval. Considering a practical constraint of limited pre-knowledge about CSI, we further transform the offline problem into an energy-aware energy efficient transmission problem. To solve the proposed problems, we undertake a convex optimization method to the optimal offline transmission scheduling problem and design corresponding optimal offline joint transmission and energy scheduling algorithm, which provides the upper bound on actual system performance. Then, we extend the non-linear fractional programming to the transmission scheduling problem with limited pre-knowledge about CSI and design corresponding joint transmission and energy scheduling algorithm, named as online algorithm. Numerical results show that the performance of the proposed online algorithm is close to that of the obtained upper bound and outperforms the existing algorithm. We also find that at each transmission time interval during the finite transmission period, the transmit power of each RRU is proportional to the weighted channel-gain-to-noise ratio (CNR) of each sub-channel.
\end{abstract}

Keywords: Energy harvesting; CoMP; Joint transmission; Energy scheduling; CSI

\section{Introduction}

The need for network operators to reduce their on-grid energy consumptions as well as carbon dioxide $\left(\mathrm{CO}_{2}\right)$ emissions is currently steering research in communications toward more efficient and environmentally friendly networks. According to [1], cellular network worldwide consumes approximately 60 billion kWh per year, among

\footnotetext{
*Correspondence: prince.lhj@gmail.com

1 State Key Laboratory of Information Security, Institute of Information Engineering, Chinese Academy of Sciences, Beijing 100093, China Full list of author information is available at the end of the article
}

which the energy consumption of base stations (BSs) accounts for $80 \%$. Fortunately, natural resources, such as wind and solar, are envisaged to be green energy resources, due to their pollution-free and renewable natures [2]. According to statistics, powering one BS which has an average power consumption of 1,400 Watts with renewable energy can reduce coal consumption of 2.5 tons with a carbon footprint of 11 tons per year [3]. Therefore, powering cellular network by renewable energy, named as renewable energy harvesting (REH) enabled cellular network in this paper, has been widely accepted as

\section{是 Springer}

(c) 2015 Wang et al: licensee Springer. This is an Open Access article distributed under the terms of the Creative Commons Attribution License (http://creativecommons.org/licenses/by/4.0), which permits unrestricted use, distribution, and reproduction in any medium, provided the original work is properly credited. 
a promising avenue to reduce $\mathrm{CO}_{2}$ emission and on-grid power consumption, e.g., the technique report [4] and the technique specification [5] of 3rd Generation Partnership Project (3GPP) clearly indicate that the use of renewable energy resources is explicitly encouraged in mobile network, and researching on it is suggested to have high priority.

On the other hand, coordinated multi-point (CoMP) transmission, which enables cooperation among multiple nodes or remote radio units (RRUs) to transmit data to the same served users with the identical time-frequency resources by the multi-antenna techniques, is regarded as a key enabling technology for elevating system performance and quality of service (QoS) [6]. Due to the promising advantage in terms of the higher spectral efficiency of its air-interface, the CoMP transmission has been accepted in the long term evolution-advanced (LTEA) specification [7].

We focus on joint transmission and harvested energy scheduling for optimizing the utility efficiency of harvested energy in hybrid energy (including on-grid energy and off-grid renewable energy) powered cellular transmission system where the off-grid REH system, mainly consisting of the energy harvester, the battery module, and the controller, is embedded into BSs.

Energy efficiency improvement is a hot topic in the research area of green wireless communication. For example, [8-10] study the energy efficient link transmission and resource allocation schemes in the orthogonal frequency division multiplexing (OFDM) system. [11] studies the energy efficient cooperative relay transmission exploiting the fractional programming approach. As for energy efficient transmission in CoMP system, [12] proposes an energy efficient power allocation algorithm for the downlink transmission, and [13] studies energy-efficient precoding for CoMP transmission in heterogeneous network.

Since the energy harvesting characteristics, i.e., the energy arrival and the amount of energy being harvested, is determined by the changing surrounding environment, e.g., the energy harvested through wind turbines varies with wind force and direction, and the energy harvested through photovoltaic panels varies with solar radiation and ambient temperature, the opportunistic energy harvesting results in a highly random energy availability. Additionally, the battery module, which can be seen as the energy buffer, provides the basis of harvested energy scheduling in the dimension of time for further improving the utilization efficiency of harvested energy. Therefore, the basic optimization problem and the optimization variables for energy efficient transmission in REH enabled cellular network are different from that in ongrid energy powered cellular network, cf., Sections 3, 4, and 5 .
Flashing back to the literature, the study of REH enabled network stems from wireless sensor network (WSN), since sensor node is often deployed under a complicated and adverse condition where a reliable on-grid power connection is not available. The short-term throughput, i.e., the amount of data transmitted during a finite time period, maximization problem is formulated in $[14,15]$. The optimal packet scheduling problem in additional white Gaussian noise (AWGN) broadcast channel is investigated in $[16,17]$. However, cellular network is more complex than WSN in terms of techniques in physical, media access control, and network layers. Besides, the energy consumption and the QoS requirement of the BS in cellular network are much higher than those of the sensor node. Therefore, it is hard to directly port the research results of REH enabled WSN to REH enabled cellular network.

As for prior studies on REH enabled cellular network, there are first some prior arts focusing on single BS transmission without considering the transmission cooperation among multiple BSs which is regarded as a promising technique for $4 \mathrm{G}$ and beyond cellular networks. In [18], an effective algorithm is proposed to maximize the utilization of green energy in such network. To maximize the utilization of green energy, an energy packet scheduler is proposed in [19] to optimize the packet scheduling. The resource allocation problem of a single cell powered by hybrid energy sources is studied in [20]. The authors analyze the relationship between energy input and transmission outage probability which is defined as the probability that the users' throughput is less than a given threshold. An energy efficient resource allocation algorithm in an OFDM access downlink network is proposed in [21].

More recently, researchers begin to draw attentions on the study of REH enabled cooperation transmission. In [22], the throughput maximization problem is investigated for only renewable energy powered relay nodes, and yet the time-varying characteristics of wireless channel are not considered. In [23], an optimal transmission scheme for a two-hop relay network is proposed under the assumption that the energy storage capacity of the battery module in REH system is infinite. In [24], a weighted sum-rate maximization problem is formulated for CoMP system powered by hybrid energy sources. Because it is assumed that there is no battery module in the energy harvesting system, the harvested energy cannot be scheduled in the dimension of time to further improve the energy efficiency of CoMP transmission and the utilization efficiency of the harvested energy. Therefore, there should be the finite energy storage module especially for the off-grid REH system.

Driven by the above considerations, the joint transmission and harvested energy scheduling problem is 
studied for a hybrid energy powered cellular network equipped with finite energy storages. Due to the random characteristic of harvested energy arrival, in such network, renewable energy is viewed as a supplement to the total amount of energy required for transmission. It is desirable to know how to sufficiently utilize renewable energy to reduce the on-grid energy consumption and the corresponding $\mathrm{CO}_{2}$ emission. Therefore, in this paper, we focus on optimizing the utilization of harvested energy, jointly considering the practical constraint of the energy harvesting system, and a priori knowledge and limited pre-knowledge about channel state information (CSI), which result in the following main contributions.

1) Considering space and cost limitations of a BS, we propose in this paper a new $\mathrm{REH}$ enabled transmission framework, in which all RRUs and associated power amplifiers (PAs) are powered by hybrid energy including on-grid energy and off-grid renewable energy, to realize a green and reliable transmission.

2) Based on the framework, we pursue a systematic study on the joint transmission and harvested energy scheduling algorithms for $\mathrm{REH}$ enabled transmission under CoMP transmission. Firstly, we formulate an optimal offline transmission scheduling problem with a priori knowledge about CSI, under constraint of available amount of harvested energy and stored energy at each transmission time interval. Then, considering practical constraint of limited pre-knowledge about CSI, we transform the optimal offline transmission scheduling problem into an energy-aware energy-efficient transmission problem.

3) We undertake a convex optimization method to the proposed optimal offline transmission scheduling problem and design corresponding optimal offline joint transmission and energy scheduling algorithm, which provide the upper bound on actual system performance. Then, we extend the non-linear fractional programming to the transmission scheduling problem with limited preknowledge about CSI and design corresponding joint transmission and energy scheduling algorithm, which named as online algorithm, considering temporal variabilities of channel and harvested energy. Numerical results show that the performance of the proposed online algorithm is close to that of the obtained upper bound. We also find that at each transmission time interval during the finite transmission time period, the transmit power of each RRU is proportional to the weighted channel-gainto-noise ratio (CNR) of each sub-channel.

The rest of the paper is organized as follows. The system model is described in Section 2. The transmission scheduling problems are formulated in Section 3. The offline solution and the proposed offline transmission scheduling algorithm are described in detail in
Section 4. Section 5 provides the online solution and the proposed online transmission scheduling algorithm. Section 6 presents the numerical results and analyses. Finally, Section 7 draws up the conclusion.

\section{System model}

As shown in Figure 1, an OFDM downlink transmission system under the green distributed BS infrastructure with a base-band unit (BBU) and $L$ RRUs is considered. Here, all the processing units (including the base-band) are pooled at BBU, and the stand-alone RRUs are installed at remote sites with only radio frequency (RF) frontend functionalities. Besides, all RRUs are connected with BBU through optical fibers. The capacity of REH system is related to the size of photovoltaic panel. Considering space and cost limitations of the BS, BBU is powered by on-grid energy for signal processing and resources allocation. Each RRU is powered by hybrid energy sources including on-grid energy source and one REH system, which consists of one energy harvester and one battery with finite-capacity $E_{C}$.

It is assumed that there are $N$ frequency sub-channels, and each of them is exclusively allocated to one user equipment (UE). Both the RRU and the UE are equipped with one single antenna, respectively. Besides, clustered CoMP transmission system is studied in this paper, by focusing on one particular cluster in which $L$ RRUs jointly transmit data to each UE, as shown in Figure 2.

The smallest time unit of resource scheduling is viewed as a time interval $[24,25]$. Consider a block fading channel model where the channel state remains static within a time interval but becomes independent across different time intervals. At each time interval, the energy arrived at each RRU is collected by energy harvester and stored in the battery before it is used for signal transmission, $\mathrm{cf}$. Figure 3.

The transmission scheduling over a finite transmission period is considered. It is assumed that there are $M+1$ time intervals during the transmission period $[0, T)$ and each with the same length, denoted by $s_{i}=\frac{T}{M+1}, \forall i \in$ $\{1,2, \ldots, M+1\}$, i.e., $s_{1}=\cdots=s_{i}=\cdots=s_{M+1}$ and $M+1$ $\sum_{i=1}^{M+1} s_{i}=T . E_{l, 0}$ denotes the initial energy available in the battery of RRU $l$ at time instant $t_{0}$. At time instants $\left\{t_{1}, t_{2}, \ldots, t_{M}\right\}$, energy arrives at RRU $l$ in amounts $\left\{E_{l, 1}, E_{l, 2}, \ldots, E_{l, M}\right\}$, as shown in Figure 4 , and can be described as

$$
E_{l, i}=\left\{\begin{array}{l}
E_{i}^{l}, \text { if } E_{i}^{l} \text { units of energy arrive at time instant } t_{i} \\
0, \text { if no energy arrives at time instant } t_{i}
\end{array}\right.
$$




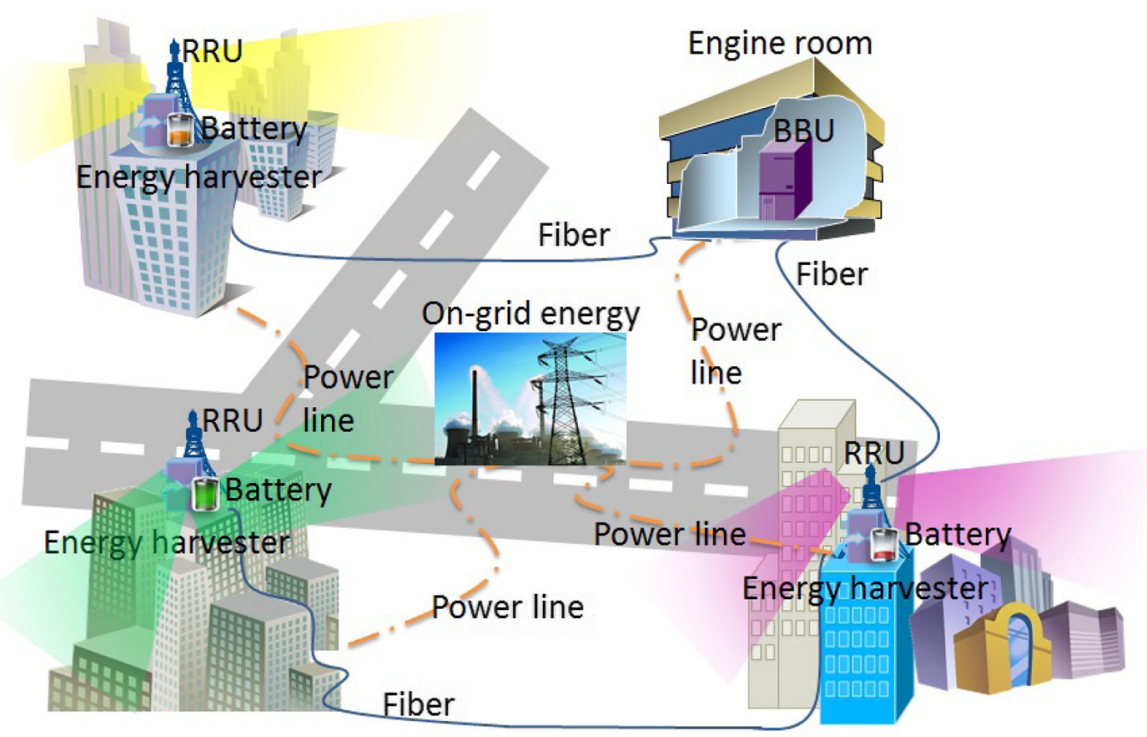

Figure 1 A downlink transmission system under the green distributed BS infrastructure with three RRUs.

where $i \in\{1,2, \ldots, M\} . E_{i}^{l}$ denotes the arrived energy of RRU $l$ at time instant $t_{i}$ and can be predicted by using renewable power prediction model, e.g., TakagiSugeno-Kang (TSK) model [26] and fuzzy inference method [27].

\section{Formulation of transmission scheduling problems}

In this section, we formulate the transmission scheduling problems for REH enabled network under CoMP transmission. We first formulate an offline transmis- sion scheduling problem with the assumption that prior knowledge about CSI is available. With consideration of practical constraint of limited pre-knowledge about CSI, we further formulate an online transmission scheduling problem by making an extension of the offline transmission scheduling problem.

\subsection{Formulation of offline transmission scheduling problem}

Each UE receives bits from $L$ RRUs which cooperate with each other. Thus, with perfect signal phase syn-

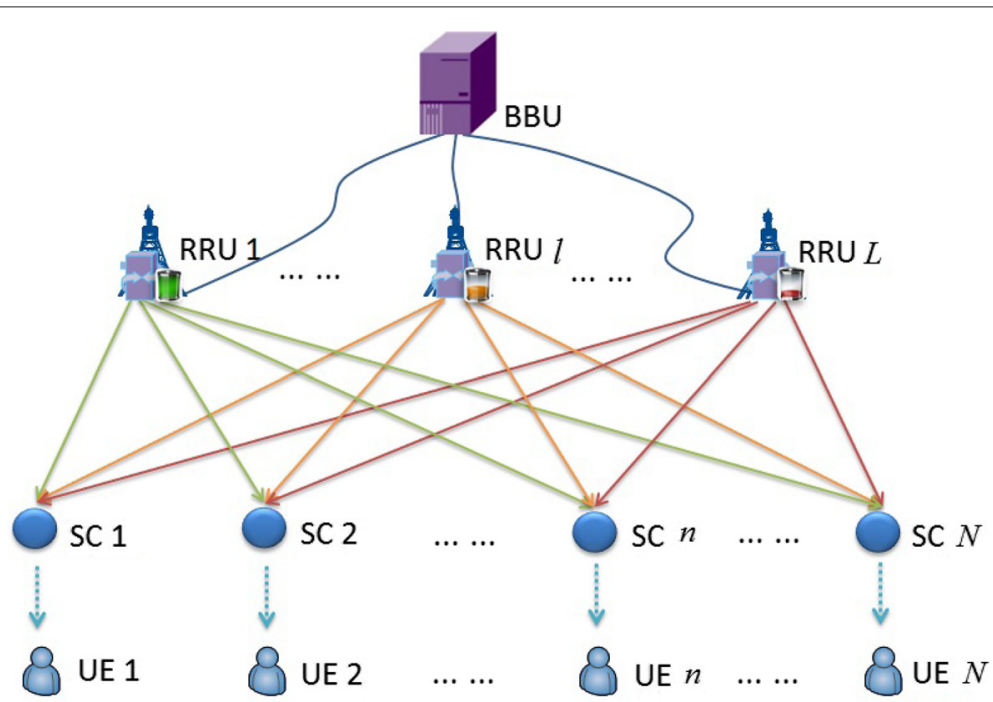

Figure 2 A L-RRU clustered CoMP transmission scenario with $N$ sub-channels. SC denotes the sub-channel. 


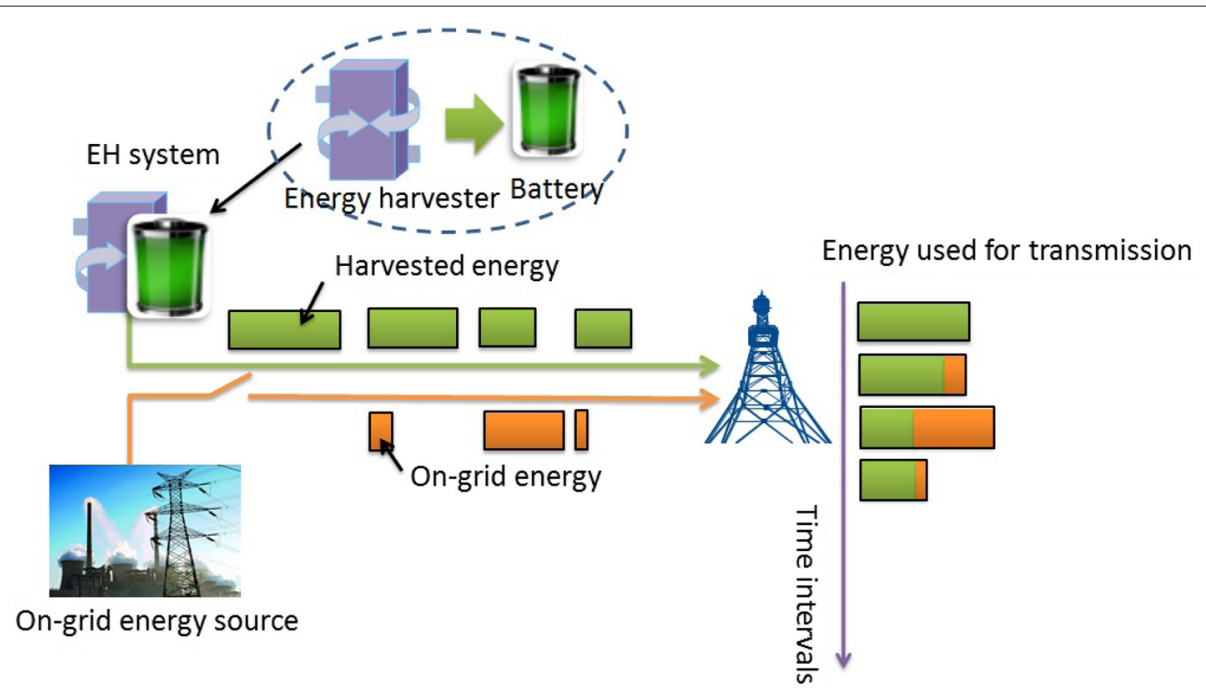

Figure 3 RRU is powered by hybrid energy source including on-grid energy source and REH system.

chronization, the achievable rate on sub-channel $n, \forall n \in$ $\{1,2, \ldots, N\}$ at the $i$ th time interval is given by

$$
R_{n, i}\left(p_{n, l, i}\right)=\log _{2}\left(1+\left(\sum_{l=1}^{L} \gamma_{n, l, i} \sqrt{p_{n, l, i}}\right)^{2}\right) .
$$

In (2), $\gamma_{n, l, i}^{2}=G_{n, l, i}\left|h_{n, l, i}\right|^{2} / \sigma^{2}$ denotes the CNR of RRU $l$ on sub-channel $n$ at the $i$ th time interval. $G_{n, l, i}$ is the large-scale path-loss from RRU $l$ on sub-channel $n$ at the $i$ th time interval and defined by $G_{n, l, i} \propto\left(d_{n, l, i} / d_{R E F}\right)^{-\alpha}$ where $d_{n, l, i}$ and $d_{R E F}$ are the distances between the UE allocated to sub-channel $n$ and the RRU $l$ at the $i$ th time interval and the reference distance, respectively. $\alpha$ is the path-loss exponent valued between 2 and $6 .\left|h_{n, l, i}\right|$ stands for the gain of the Rayleigh fading channel at the $i$ th time interval, modeled by i.i.d. complex Gaussian with unit variance, i.e., $h_{n, l, i} \sim \mathcal{N}_{C}(0,1) . \sigma^{2}$ is the power of AWGN. Without loss of generality, it is assumed that the noise power is the same for all the sub-channels. $p_{n, l, i}$ is the transmit power of RRU $l$ on sub-channel $n$ at the $i$ th time interval.
To maximize the number of bits transmitted during the considered transmission period $[0, T)$, the following optimization problem is formulated.

$$
\begin{aligned}
& \max _{p_{n, l, i}} \sum_{n=1}^{N} \sum_{i=1}^{M+1} R_{n, i}\left(p_{n, l, i}\right) s_{i} \\
& \text { s.t. } \mathrm{C} 1: \sum_{i=1}^{m} E_{l, i}^{c o n} \geq\left(\sum_{i=0}^{m} E_{l, i}-E_{C}\right)^{+}, \\
& \mathrm{C} 2: \sum_{i=1}^{m^{\prime}} E_{l, i}^{c o n} \leq \sum_{i=0}^{m^{\prime}-1} E_{l, i},
\end{aligned}
$$

where $m \in\{1,2, \ldots, M\}, m^{\prime} \in\{1,2, \ldots, M+1\}$ and $l \in\{1,2, \ldots, L\} . E_{l, i}^{c o n}=\left(\xi \sum_{n=1}^{N} p_{n, l, i}+P_{C}\right) s_{i}$ denotes the energy consumption of RRU $l$ at the $i$ th time interval. $\xi$ is defined as $\xi=\varepsilon / \eta$, where $\varepsilon$ denotes the peak-to-average power ratio (PAPR) and $\eta$ denotes the PA efficiency. $P_{C}$

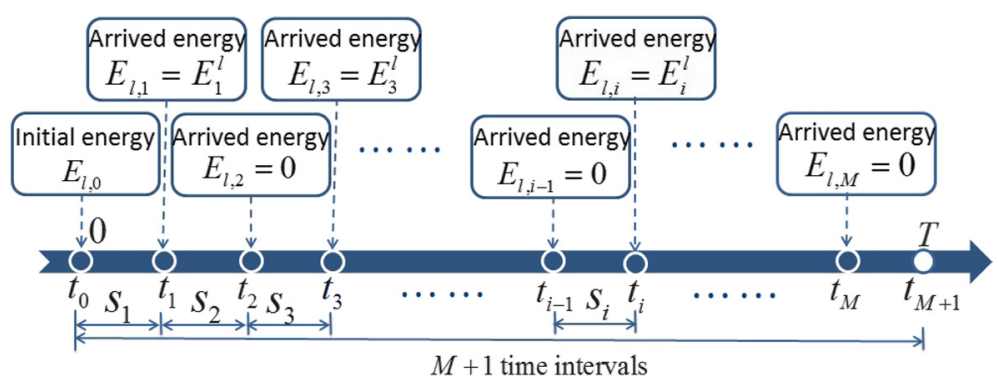

Figure 4 The sequences of energy arrivals and time intervals. 
stands for the power consumption of other facilities in the RRU. $(\cdot)^{+}$is defined as $(\cdot)^{+}=\max \{0, \cdot\}$. Non-overflow constraint $\mathrm{C} 1$ states that in order to prevent energy overflow in the battery of RRU $l, \forall l \in\{1,2, \ldots, L\}$, at least $\left(\sum_{i=0}^{m} E_{l, i}-E_{C}\right)^{+}$amount of energy must be consumed by the time the $m$ th energy arrives. In other words, the total amount of energy stored in the battery of each RRU never exceeds the battery capacity $E_{C}$. Causality constraint $\mathrm{C} 2$ implies that during every transmission period $\left[0, t_{m}\right), \forall m \in\{1,2, \ldots, M+1\}$, the energy that can be drawn from the battery of RRU $l$ to cover the energy requirement at the $\mathrm{PA}$ is constrained to consume at most the amount of available energy currently stored in the battery [21]. Which means that even more energy will be harvested in future time but can not be used to meet the energy requirement currently.

\subsection{Formulation of online transmission scheduling problem}

So far in our formulation, we have considered the offline problem with the assumption that a priori knowledge about CSI during the whole transmission period $[0, T)$ is available. However, in practice, it is difficult to obtain the prior knowledge about CSI during the whole transmission period $[0, T)[28]$. Fortunately, with channel prediction technology which has been extensively investigated in the literature $[29,30]$, it is possible to obtain the predicted CSI for a finite time period. Specifically, we further formulate the transmission scheduling problem in an online manner by assuming the knowledge about the predicted CSI for each two consecutive time intervals.

The online problem is formulated in two steps, and details are given as follows.

Step 1: With the help of $E_{t o t}=\sum_{l=1}^{L} \sum_{i=0}^{M+1} E_{l, i}$, denoting the total available energy of the system during the whole transmission period $[0, T)$, the objective function of optimization problem (3) can be described as

$$
\begin{aligned}
\max & \sum_{n=1}^{N} \sum_{i=1}^{M+1} R_{n, i}\left(p_{n, l, i}\right) s_{i} \\
=\max & \frac{\sum_{n=1}^{N} \sum_{i=1}^{M+1} R_{n, i}\left(p_{n, l, i}\right) s_{i}}{E_{t o t}} \cdot E_{t o t} \\
=\max & \bar{U}_{E E} \cdot E_{t o t},
\end{aligned}
$$

where $\bar{U}_{E E}$ denotes average energy efficiency, measured in bit/Joule. Hence, with the prior knowledge about CSI during the whole transmission period [0,T), to maximize the total number of transmitted bits is to maximize the average energy efficiency of the system.

Step 2: We assume that the knowledge about the predicted CSI during the time period $\left[t_{k-1}, t_{k+1}\right), \forall k \in$ $\{1,2, \ldots, M\}$, is available, i.e., the $k$ th and $k+1$ th time intervals. Therefore, based on Step 1, for any transmission period $\left[0, t_{k+1}\right), \quad \forall k \in\{1,2, \ldots, M\}$, it is reasonable to regard the average energy efficiency maximization problem during $\left[0, t_{k+1}\right)$ with predicted CSI during $\left[t_{k-1}, t_{k+1}\right)$ as the transmission scheduling target, which can be formulated as

$$
\begin{gathered}
\max _{\widehat{p}_{n, l, i}} \frac{\sum_{n=1}^{N} \sum_{i=1}^{k-1} B_{n, i}^{\prime}+\sum_{n=1}^{N} \sum_{i=k}^{k+1} R_{n, i}\left(\widehat{p}_{n, l, i}\right) s_{i}}{\sum_{l=1}^{L} \sum_{i=1}^{k-1} E_{l, i}^{\prime}+\sum_{l=1}^{L} \sum_{i=k}^{k+1} E_{l, i}^{c o n}} \\
\text { s.t. } \mathrm{C} 3: \sum_{i=1}^{k-1} E_{l, i}^{\prime}+\sum_{i=k}^{k+1} E_{l, i}^{c o n} \geq\left(\sum_{i=0}^{k+1} E_{l, i}-E_{C}\right)^{+}, \\
\text {C4 : } \sum_{i=1}^{k-1} E_{l, i}^{\prime}+\sum_{i=k}^{u} E_{l, i}^{c o n} \leq \sum_{i=0}^{u-1} E_{l, i} .
\end{gathered}
$$

In (5), $n \in\{1,2, \ldots, N\}, l \in\{1,2, \ldots, L\}$ and $u \in\{k, k+1\} . B_{n, i}^{\prime}$ is the bits transmitted on subchannel $n$ at the $i$ th time interval and given by $B_{n, i}^{\prime}=$ $\log _{2}\left(1+\left(\sum_{l=1}^{L} \gamma_{n, l, i} \sqrt{\widehat{p}_{n, l, i}}\right)^{2}\right) s_{i}$, where $\widehat{p}_{n, l, i}$ is the transmit power of RRU $l$ on sub-channel $n$ at the $i$ th time interval. $E_{l, i}^{\prime}=\left(\xi \sum_{n=1}^{N} \widehat{p}_{n, l, i}+P_{C}\right) s_{i}$ denotes the energy consumed by RRU $l$ at the $i$ th time interval. $\left\{B_{n, i}^{\prime}\right\}$ and $\left\{E_{l, i}^{\prime}\right\}$ are constant sequences which have been determined by resolving the above optimization problem during the transmission period $\left[0, t_{i+1}\right), \quad \forall i \in\{1,2, \ldots, k-1\}$. Note that in (5), $\sum_{i=1}^{k-1} B_{n, i}^{\prime}$ and $\sum_{i=1}^{k-1} E_{l, i}^{\prime}$ are equal to zero when $k=1$ for $\forall n$.

The transmission scheduling with limited preknowledge about CSI is obtained by solving (5) for each two consecutive time intervals, as is shown in Figure 5. The grey part denotes the transmission period during which the bits can be transmitted, and the consumed energy for each of the time intervals has been determined, i.e., the transmission scheduling has been determined. The time period framed by the red box denotes the time intervals at which the transmission scheduling is about to be determined with predicted CSI at the current and the next time intervals. 


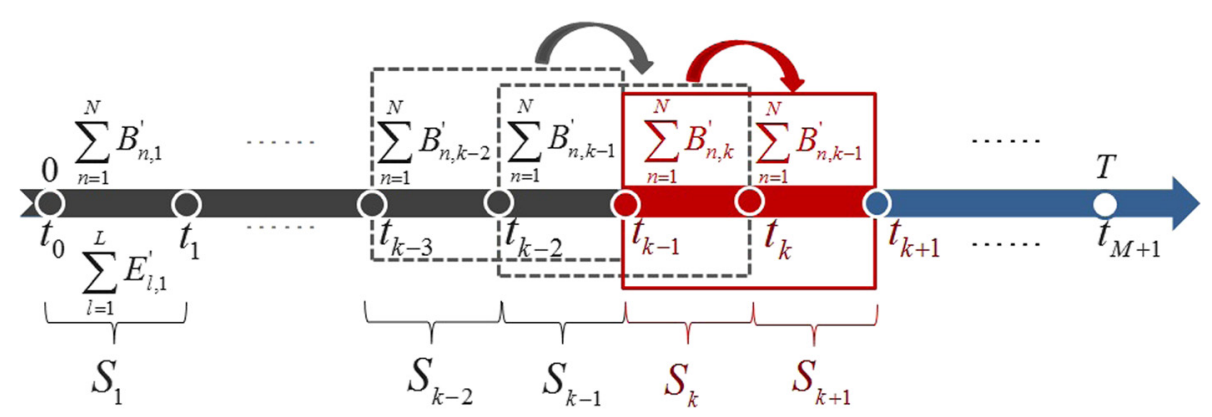

Figure 5 A graphic explaining the online transmission scheduling problem.

\section{Offline solution and joint transmission and energy scheduling algorithm}

The offline optimization problem (3) can be proved to be concave with the composition rules of concavity and the first order condition [31], with details as follows.

Proof: Making $D\left(\left\{p_{n, l, i}\right\}\right)=\sum_{n=1}^{N} \sum_{i=1}^{M+1} R_{n, i}\left(p_{n, l, i}\right) s_{i}=$ $\sum_{n=1}^{N} \sum_{i=1}^{M+1} D_{n, i} s_{i}$ where

$$
\begin{aligned}
D_{n, i} & =\log _{2}\left(1+\left(\sum_{l=1}^{L} \gamma_{n, l, i} \sqrt{p_{n, l, i}}\right)^{2}\right) \\
& =\log _{2}\left(1+\sum_{l=1}^{L} \gamma_{n, l, i}^{2} p_{n, l, i}+\sum_{l=1}^{L} \sum_{k \neq l} \gamma_{n, l, i} \gamma_{n, k, i} \sqrt{p_{n, l, i} p_{n, k, i}}\right) .
\end{aligned}
$$

Since $D\left(\left\{p_{n, l, i}\right\}\right)$ is a linear combination of $D_{n, i}$, $D\left(\left\{p_{n, l, i}\right\}\right)$ is concave if $D_{n, i}$ is concave.

$$
\text { Making } \mathrm{G}=\log _{2}(\cdot), f_{n, i}=\sum_{l=1}^{L} \gamma_{n, l, i}^{2} p_{n, l, i} \text { and } g_{n, i}=
$$

$\sum_{l=1}^{L} \sum_{k \neq l} \gamma_{n, l, i} \gamma_{n, k, i} \sqrt{p_{n, l, i} p_{n, k, i}}$, then $D_{n, i}$ can be written as

$$
D_{n, i}=G\left(1+f_{n, i}+g_{n, i}\right)
$$

where $n \in\{1,2, \ldots, N\}$ and $i \in\{1,2, \ldots, M+1\}$, respectively.

Since $\log _{2}(\cdot)$ is concave and non-decreasing, and $f_{n, i}$ is linear, $D_{n, i}$ is concave if $g_{n, i}$ is concave according to the composition rules of convexity [31]. The concavity of $g_{n, i}$ is verified with the first order condition [31].
Making $\mathbf{x}_{\mathbf{1}}=\left[p_{k, 1, i}^{x_{1}}, p_{k, 2, i}^{x_{1}}, \ldots, p_{k, L, i}^{x_{1}},\right]^{T}$ and $\mathbf{x}_{2}=$ $\left[p_{k, 1, i}^{x_{2}}, p_{k, 2, i}^{x_{2}}, \ldots, p_{k, L, i}^{x_{2}},\right]^{T}$, then we have

$$
\begin{aligned}
& g_{n, i}\left(\mathbf{x}_{\mathbf{1}}\right)-g_{n, i}\left(\mathbf{x}_{2}\right)-\nabla g_{n, i}\left(\mathbf{x}_{\mathbf{2}}\right)^{T}\left(\mathbf{x}_{\mathbf{1}}-\mathbf{x}_{\mathbf{2}}\right) \\
& =\sum_{k \neq l}\left(\begin{array}{l}
\sqrt{p_{n, l, i}^{x_{1}} p_{n, k, i}^{x_{1}}}-p_{n, l, i}^{x_{1}} \sqrt{\frac{p_{n, k, i}^{x_{2}}}{p_{n, l, i}^{x_{2}}}} \\
\left.-p_{n, l, i}^{x_{1}} \sqrt{\frac{p_{n, l, i}^{x_{2}}}{p_{n, k, i}^{x_{2}}}}\right)^{2}
\end{array}\right) \gamma_{n, l, i} \gamma_{n, k, i} \\
& =-\sum_{k \neq l}\left(\begin{array}{l}
\sqrt{p_{n, l, i}^{x_{1}}}\left(\frac{p_{n, k, i}^{x_{2}}}{p_{n, l, i}^{x_{2}}}\right)^{\frac{1}{4}} \\
-\sqrt{p_{n, l, i}^{x_{1}}}\left(\frac{p_{n, l, i}^{x_{2}}}{p_{n, k, i}^{x_{2}}}\right)^{\frac{1}{4}}
\end{array}\right)^{2} \gamma_{n, l, i} \gamma_{n, k, i} \leq 0,
\end{aligned}
$$

i.e. $g_{n, i}\left(\mathbf{x}_{\mathbf{1}}\right)-g_{n, i}\left(\mathbf{x}_{2}\right) \leq \nabla g_{n, i}\left(\mathbf{x}_{2}\right)^{T}\left(\mathbf{x}_{\mathbf{1}}-\mathbf{x}_{2}\right)$.

Hence, $g_{n, i}$ is concave and consequently, $D\left(\left\{p_{n, l, i}\right\}\right)$ is concave.

Therefore, we can solve the maximization problem (3) with dual decomposition. Upon rearranging terms, the Lagrangian associated with the problem (3) can be written as

$$
\begin{aligned}
\mathcal{L}\left(\varsigma_{l, m}, \mu_{l, m^{\prime}}, p_{n, l, i}\right) & \\
= & \sum_{l=1}^{L} \sum_{m=1}^{M} \varsigma_{l, m}\left(\sum_{i=1}^{m} E_{l, i}^{c o n}-\left(\sum_{i=0}^{m} E_{l, i}-E_{C}\right)^{+}\right) \\
& +\sum_{l=1}^{L} \sum_{m^{\prime}=1}^{M+1} \mu_{l, m^{\prime}}\left(\sum_{i=0}^{m^{\prime}-1} E_{l, i}-\sum_{i=1}^{m^{\prime}} E_{l, i}^{c o n}\right) \\
& +\sum_{n=1}^{N} \sum_{i=1}^{M+1} R_{n, i}\left(p_{n, l, i}\right) s_{i}
\end{aligned}
$$

where $\left\{\varsigma_{l, m}\right\}$ and $\left\{\mu_{l, m^{\prime}}\right\}$ denote the Lagrange multiplier matrices associated with constraints $\mathrm{C} 1$ and $\mathrm{C} 2$, respectively. 
Thus, the dual problem is given by

$$
\min _{\varsigma_{l, m}, \mu_{l, m^{\prime}} \geq 0} \max _{p_{n, l, i}} \mathcal{L}\left(\varsigma_{l, m}, \mu_{l, m^{\prime}}, p_{n, l, i}\right) .
$$

Making the derivative of $\mathcal{L}\left(\varsigma_{l, m}, \mu_{l, m^{\prime}}, p_{n, l, i}\right)$ in (9) respecting to $p_{n, l, i}$ equal to zero, we obtain

$$
\frac{\partial \mathcal{L}\left(\varsigma_{l, m}, \mu_{\left.l, m^{\prime}, p_{n, l, i}\right)}\right.}{\partial p_{n, l, i}}=\frac{s_{i} W_{n, i} \gamma_{n, l, i}}{\sqrt{p_{n, l, i}} \ln 2}-\mathcal{K}_{l, i} \xi s_{i}=0,
$$

where

$$
W_{n, i}=\frac{\sum_{g=1}^{L} \gamma_{n, g, i} \sqrt{p_{n, g, i}}}{1+\left(\sum_{g=1}^{L} \gamma_{n, g, i} \sqrt{p_{n, g, i}}\right)^{2}},
$$

and

$$
\mathcal{K}_{l, i}=\sum_{m^{\prime}=i}^{M+1} \mu_{l, m^{\prime}}-\sum_{m=i}^{M} \varsigma_{l, m}
$$

Define $\varphi_{n, i}=\left\{l \mid p_{n, l, i}>0, l=1,2, \ldots, L\right\}, \quad \forall n \quad \in$ $\{1,2, \ldots, N\}$ as the set of RRUs which have the positive transmit power on sub-channel $n$ at the $i$ th time interval. According to (11), there is

$$
\begin{aligned}
\frac{\gamma_{n, l_{1}, i}}{\mathcal{K}_{l_{1}, i} \sqrt{p_{n, l_{1}, i}}} & =\cdots=\frac{\gamma_{n, l_{\vartheta}, i}}{\mathcal{K}_{l_{\vartheta}, i} \sqrt{p_{n, l_{\vartheta}, i}}} \\
& =\cdots=\frac{\gamma_{n, l_{L_{n}, i}}}{\mathcal{K}_{l_{L_{n}}, i} \sqrt{p_{n, l_{L_{n}}, i}}}=\frac{\xi \ln 2}{W_{n, i}},
\end{aligned}
$$

where $\vartheta \in\left\{1,2, \ldots, L_{n}\right\}$ and $l_{1}, \ldots, l_{L_{n}} \in \varphi_{n, i} . L_{n}=\left|\varphi_{n, i}\right|$ is the size of $\varphi_{n, i}$. Therefore, $p_{n, l_{\vartheta}, i}$ can be derived as

$$
\sqrt{p_{n, l_{\vartheta}, i}}=\frac{\gamma_{n, l_{\vartheta}, i}}{\gamma_{n, l_{1}, i}} \cdot \frac{\mathcal{K}_{l_{1}, i}}{\mathcal{K}_{l_{\vartheta}, i}} \cdot \sqrt{p_{n, l_{1}, i}},
$$

where $\mathcal{K}_{l_{1}, i} \cdot \gamma_{n, l_{\vartheta}, i}$ can be viewed as the weighted CNR of RRU $l_{\vartheta}$.

Substituting (15) into (11) and (12), and making $p_{n, l, i}=$ $0, \forall l \notin \varphi_{n, i}$, the optimal solution of (3) can be expressed as

$$
p_{n, l, i}^{*}= \begin{cases}\frac{1}{H_{n, l, i} \mathcal{K}_{l, i}^{2}}\left(\frac{\sum_{g \in \varphi_{n, i}^{*}} \frac{\gamma_{n, g, i}^{2}}{\kappa_{g, i}}}{\xi \ln 2}-1\right)^{+}, l \in \varphi_{n, i}^{*} \\ 0 \quad, l \notin \varphi_{n, i}^{*}\end{cases}
$$

where $\varphi_{n, i}^{*}=\left\{l \mid p_{n, l, i}^{*}>0, l=1,2, \ldots, L\right\}$ and

$$
H_{n, l, i}=\frac{\left(\sum_{g \in \varphi_{n, i}^{*}} \frac{\gamma_{n, g, i}^{2}}{\mathcal{K}_{g, i}}\right)^{2}}{\gamma_{n, l, i}^{2}} .
$$

According to (16), the optimal $\varphi_{n, i}^{*}$ need to be selected, which may need to search over $2^{L}$ cases for each time interval. However, we will prove in following that there are only two possible cases for $\varphi_{n, i}^{*}$. To describe our theory, two lemmas are first given as follows.
Lemma 1: The solution satisfying (16) with all $p_{n, l, i}^{*}>$ 0 , i.e., $\varphi_{n, i}^{*}=\{1,2, \ldots, L\}$, is an extreme point solution. Moreover, it is also the optimal solution of (3), if exists, due to the concavity of the objective function in (3).

Lemma 2: The solutions satisfying (16) with at least one $p_{n, l, i}^{*}=0$, i.e., $\varphi_{n, i}^{*} \subset\{1,2, \ldots, L\}$, are boundary point solutions. When the extreme point is not feasible, the optimal solution must be one of these solutions.

To solve the optimization problem (3), it is natural that to first check whether the extreme point is feasible. If not, then check each boundary point solution.

With the above two lemmas, the following theorem is provided.

Theorem 1: If the extreme point solution does not exist, then the feasible solution of (3) must be the solution with all $p_{n, l, i}^{*}=0$, i.e., $\varphi_{n, i}^{*}=\emptyset$.

Proof: According to (16), if the extreme point solution does not exist, there is

$$
p_{n, l, i}^{*}<0 \Leftrightarrow \sum_{g \in \varphi_{n, i}^{*}} \frac{\gamma_{n, g, i}^{2}}{\mathcal{K}_{g, i}}<\xi \ln 2,
$$

where $\varphi_{n, i}^{*}=\{1,2, \ldots, L\}$.

Supposing there exist a boundary point solution $\left\{p_{n, l, i}^{\prime}\right\}$ satisfying (16) with $\varphi_{n, i}^{\prime}=\left\{l \mid p_{n, l, i}^{\prime}>0, l=1,2, \ldots, L\right\} \neq$ $\emptyset$ and $\varphi_{n, i}^{\prime} \subset \varphi_{n, i}^{*}$, then

$$
\sum_{g \in \varphi_{n, i}^{\prime}} \frac{\gamma_{n, g, i}^{2}}{\mathcal{K}_{g, i}}<\sum_{g \in \varphi_{n, i}^{*}} \frac{\gamma_{n, g, i}^{2}}{\mathcal{K}_{g, i}}<\xi \ln 2 \Leftrightarrow p_{n, l, i}^{\prime}<0, l \in \varphi_{n, i}^{\prime}
$$

i.e., the solution $\left\{p_{n, l, i}^{\prime}\right\}$ is not feasible. Hence, except the solution with $p_{n, l, i}^{*}=0$ and $\varphi_{n, i}^{*}=\emptyset$, none of the boundary point solutions is feasible.

Therefore, for the optimal solution of (3) in (16), $\varphi_{n, i}^{*}$ equals either $\{1,2, \ldots, L\}$ or $\emptyset$. The optimal solution of (3) can be reformed as

$$
p_{n, l, i}^{*}=\frac{1}{H_{n, l, i} \mathcal{K}_{l, i}^{2}}\left(\frac{\sum_{l=1}^{L} \frac{\gamma_{n, l, i}^{2}}{\mathcal{K}_{l, i}}}{\xi \ln 2}-1\right)^{+}
$$

where $\forall n \in\{1,2, \ldots, N\}, \forall l \in\{1,2, \ldots, L\}$ and $\forall i \in$ $\{1,2, \ldots, M+1\}$. 
Table 1 Simulation parameters

\begin{tabular}{lc}
\hline Simulation parameter & Value \\
\hline Number of RRUs $L$ & 2 \\
Number of sub-channels $N$ & 12 \\
Number of time intervals $M+1$ & 10 \\
Length of each time interval $s_{i}(\mathrm{~s})$ & 1 \\
Battery capacity $E_{C}(J)$ & 300 \\
Static circuit power consumption $P_{C}(\mathrm{dBm})$ & 40 \\
Power amplifier efficiency $\eta$ & 0.3 \\
Peak-to-average power ratio $\varepsilon(\mathrm{dB})$ & 12 \\
Path-loss exponent $\alpha$ & 4 \\
Noise power $\sigma^{2}(\mathrm{dBm})$ & -128 \\
Reference distance $d_{R E F}(\mathrm{~m})$ & 35 \\
\hline
\end{tabular}

The gradient update equations for $\left\{\varsigma_{l, m}\right\}$ and $\left\{\mu_{l, m^{\prime}}\right\}$ are given as

$$
\begin{gathered}
\varsigma_{l, m}^{v+1}=\left(\varsigma_{l, m}^{v}-\kappa_{1}^{v}\left(\sum_{i=1}^{m} E_{l, i}^{c o n}-\left(\sum_{i=0}^{m} E_{l, i}-E_{C}\right)^{+}\right)\right)^{+}, \\
\mu_{l, m^{\prime}}^{v+1}=\left(\mu_{l, m^{\prime}}^{v}-\kappa_{2}^{v}\left(\sum_{i=0}^{m^{\prime}-1} E_{l, i}-\sum_{i=1}^{m^{\prime}} E_{l, i}^{c o n}\right)\right)^{+}
\end{gathered}
$$

where $l \in\{1,2, \ldots, L\} ; m \in\{1,2, \ldots, M\} ; m^{\prime} \in$ $\{1,2, \ldots, M+1\} ; v$ is the iteration number; $\kappa_{1}^{v}$ and $\kappa_{2}^{v}$ are the sequences of scalar step size.

\section{Online solution and joint transmission and energy scheduling algorithm}

The optimization problem in (5) is a nonlinear fractional programming problem [30] and can be associ- ated with the following parametric programming (PP) problem.

$$
\begin{aligned}
\max _{\widehat{p}_{n, l, i}} & \left(\left(\sum_{n=1}^{N} \sum_{i=1}^{k-1} B_{n, i}^{\prime}+\sum_{n=1}^{N} \sum_{i=k}^{k+1} R_{n, i}\left(\widehat{p}_{n, l, i}\right) s_{i}\right)\right. \\
& \left.-q\left(\sum_{l=1}^{L} \sum_{i=1}^{k-1} E_{l, i}^{\prime}+\sum_{l=1}^{L} \sum_{i=k}^{k+1} E_{l, i}^{c o n}\right)\right) .
\end{aligned}
$$

In (23), $q \in R$ is referred as a parameter which determines the relative weight of the total energy consumption of system and can be intuitively interpreted as the overhead caused by energy consumption [32].

We denote the optimal solution $\widehat{p}_{n, l, i}$ in (23) as $\widehat{p}_{n, l, i}^{*}$ with a fixed $q$. According to Dinkelbach's theory [33], when $\sum_{n=1}^{N} \sum_{i=1}^{k-1} B_{n, i}^{\prime}+\sum_{n=1}^{N} \sum_{i=k}^{k+1} R_{n, i}\left(\widehat{p}_{n, l, i}\right) s_{i}$ is concave, and $\sum_{l=1}^{L} \sum_{i=1}^{k-1} E_{l, i}^{\prime}+\sum_{l=1}^{L} \sum_{i=k}^{k+1} E_{l, i}^{c o n}$ is convex, the $\widehat{p}_{n, l, i}^{*}$ is also optimal for (5) if, and only if,

$$
\begin{aligned}
& \sum_{n=1}^{N} \sum_{i=1}^{k-1} B_{n, i}^{\prime}+\sum_{n=1}^{N} \sum_{i=k}^{k+1} R_{n, i}\left(\widehat{p}_{n, l, i}^{*}\right) s_{i} \\
& -q\left(\sum_{l=1}^{L} \sum_{i=1}^{k-1} E_{l, i}^{\prime}+\sum_{l=1}^{L} \sum_{i=k}^{k+1} E_{l, i}^{c o n}\right)=0 .
\end{aligned}
$$

and the corresponding $q$ denotes the optimal average energy efficiency in (5).

Therefore, the Dinkelbach's theory indicates a possible method to solve the problem (5). For the transmission period $\left[0, t_{k+1}\right),\left\{B_{n, i}^{\prime}\right\}$ and $\left\{E_{l, i}^{\prime}\right\}, \forall i \in\{1,2, \ldots, k-1\}$, are constant sequences mentioned earlier. The proof of
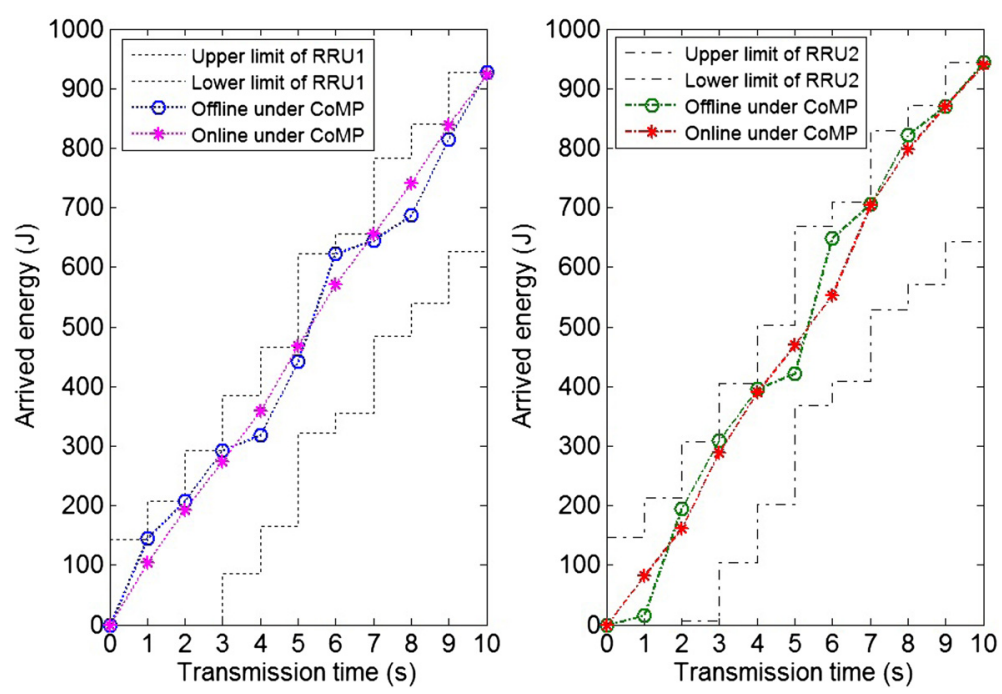

Figure 6 Accumulated energy consumption versus transmission time. 


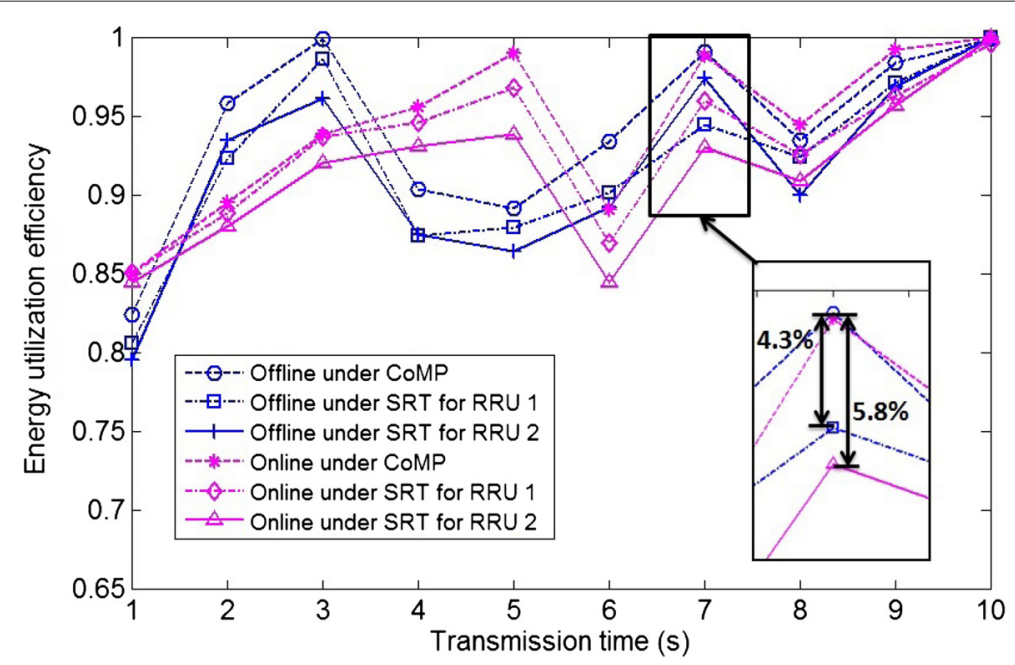

Figure 7 Energy utilization efficiency versus transmission time.

concavity of $\sum_{n=1}^{N} \sum_{i=k}^{k+1} R_{n, i}\left(\widehat{p}_{n, l, i}\right) s_{i}$ is similar to the proof of concavity of the objective function of offline problem. $\sum_{i=k}^{k+1} E_{l, i}^{c o n}$ is a linear function of $\widehat{p}_{n, l, i}$, which satisfies the convexity. Hence, the primal problem in (5) is equivalent with the following optimization problem.

$$
\begin{array}{r}
\max _{\widehat{p}_{n, l, i}}\left(\sum_{n=1}^{N} \sum_{i=1}^{k-1} B_{n, i}^{\prime}+\sum_{n=1}^{N} \sum_{i=k}^{k+1} R_{n, i}\left(\widehat{p}_{n, l, i}\right) s_{i}\right. \\
\left.-q\left(\sum_{l=1}^{L} \sum_{i=1}^{k-1} E_{l, i}^{\prime}+\sum_{l=1}^{L} \sum_{i=k}^{k+1} E_{l, i}^{c o n}\right)\right)
\end{array}
$$

The Lagrangian of (25) is given by

$$
\mathcal{L}\left(\rho_{l}, \psi_{l, u}, \widehat{p}_{h, l, i}\right)
$$

$$
\begin{aligned}
= & \sum_{l=1}^{L} \rho_{l}\left(\sum_{i=1}^{k-1} E_{l, i}^{\prime}+\sum_{i=k}^{k+1} E_{l, i}^{c o n}-\left(\sum_{i=0}^{k+1} E_{l, i}-E_{C}\right)^{+}\right) \\
& +\sum_{l=1}^{L} \sum_{u=k}^{k+1} \psi_{l, u}\left(\sum_{i=0}^{u-1} E_{l, i}-\left(\sum_{i=1}^{k-1} E_{l, i}^{\prime}+\sum_{i=k}^{u} E_{l, i}^{c o n}\right)\right) \\
& +\sum_{n=1}^{N} \sum_{i=1}^{k-1} B_{n, i}^{\prime}+\sum_{n=1}^{N} \sum_{i=k}^{k+1} R_{n, i}\left(\widehat{p}_{l, l, i}\right) s_{i} \\
& -q\left(\sum_{l=1}^{L} \sum_{i=1}^{k-1} E_{l, i}^{\prime}+\sum_{l=1}^{L} \sum_{i=k}^{k+1} E_{l, i}^{c o n}\right),
\end{aligned}
$$

s.t. $\mathrm{C} 3, \mathrm{C} 4$.

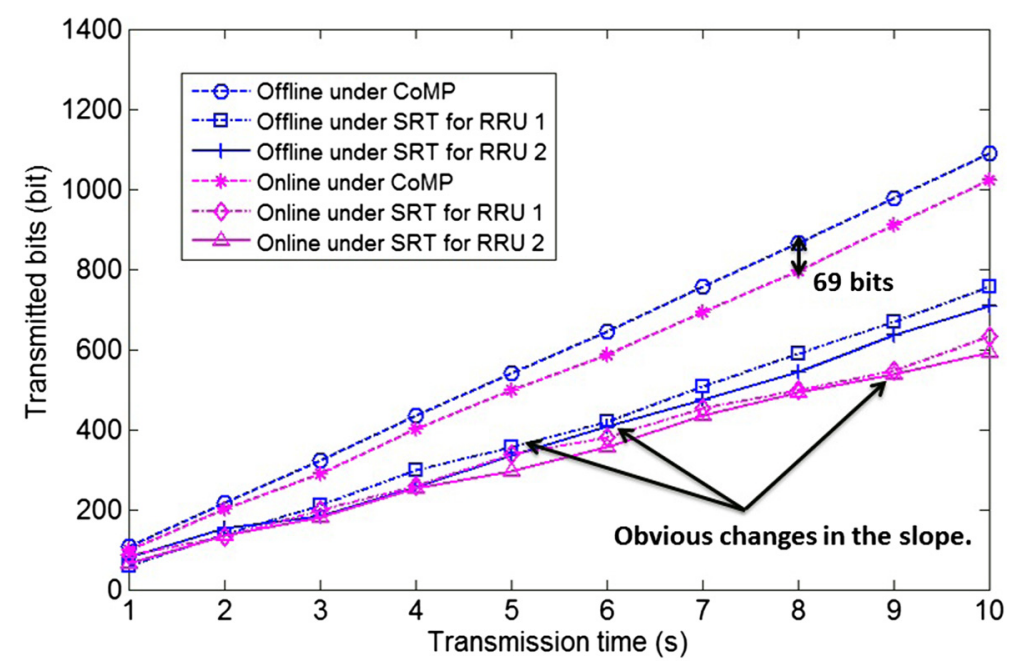

Figure 8 Transmitted bits versus transmission time. 
where $\left\{\rho_{l}\right\}$ and $\left\{\psi_{l, u}\right\}$ denote the Lagrange multiplier vector and matrix associated with constraint C3 and constraint C4, respectively.

Thus, the dual optimization problem is given by

$$
\min _{\rho_{l}, \psi_{l, u} \geq 0} \max _{\widehat{p}_{n, l, i}} \mathcal{L}\left(\rho_{l}, \psi_{l, u}, \widehat{p}_{n, l, i}\right)
$$

By using dual decomposition and following a similar approach as in (11) - (20), the optimal solution of (5) with a fixed $q$ can be described as

$$
\widehat{p}_{n, l, i}^{*}=\frac{1}{\bar{H}_{n, l, i} \overline{\mathcal{K}}_{l, i}^{2}}\left(\frac{\sum_{l=1}^{L} \frac{\gamma_{n, l, i}^{2}}{\overline{\mathcal{K}}_{l, i}}}{\xi \ln 2}-1\right)^{+}
$$

where $\forall n \in\{1,2, \ldots, N\}, \forall l \in\{1,2, \ldots, L\}$ and $\forall i \in\{k, k+$ $1\} . \overline{\mathcal{K}}_{l, i}$ is given by

$$
\overline{\mathcal{K}}_{l, i}= \begin{cases}\sum_{u=k}^{k+1} \psi_{l, u}-\rho_{l}+q, & i=k \\ \psi_{l, k+1}+q & , i=k+1\end{cases}
$$

and $\bar{H}_{n, l, i}$ is described as

$$
\bar{H}_{n, l, i}=\frac{\left(\sum_{g=1}^{L} \frac{\gamma_{n, g, i}^{2}}{\overline{\mathcal{K}}_{g, i}}\right)^{2}}{\gamma_{n, l, i}^{2}} .
$$

Thus, the power allocated at the $k$ th time interval for signal transmission of RRU $l$ on sub-channel $n$ is determined by $\widehat{p}_{n, l, k}^{*}=\left\{\widehat{p}_{n, l, i}^{*} \mid n \in\{1,2, \ldots, N\}, l \in\{1,2, \ldots, L\}\right.$, $i=k\}$.

The gradient update equations for $\left\{\rho_{l}\right\}$ and $\left\{\psi_{l, u}\right\}$ are given as

$$
\rho_{l}^{v+1}=\left(\rho_{l}^{v}-\kappa_{3}^{v}\left(\sum_{i=1}^{k-1} E_{l, i}^{\prime}+\sum_{i=k}^{k+1} E_{l, i}^{c o n}-\left(\sum_{i=0}^{k+1} E_{l, i}-E_{C}\right)^{+}\right)\right)^{+},
$$

$$
\psi_{l, u}^{v+1}=\left(\psi_{l, u}^{v}-\kappa_{4}^{v}\left(\sum_{i=0}^{u-1} E_{l, i}-\sum_{i=1}^{k-1} E_{l, i}^{\prime}-\sum_{i=k}^{u} E_{l, i}^{c o n}\right)\right)^{+}
$$

where $l \in\{1,2, \ldots, L\} ; u \in\{k, k+1\} ; v$ is the iteration number; $\kappa_{3}^{v}$ and $\kappa_{4}^{v}$ are the sequences of scalar step size.

The proposed offline and online transmission scheduling algorithms are summarized in Algorithm 1 and Algorithm 2, respectively. The proofs of convergence of proposed offline and online algorithms are similar to Appendix B in [34].
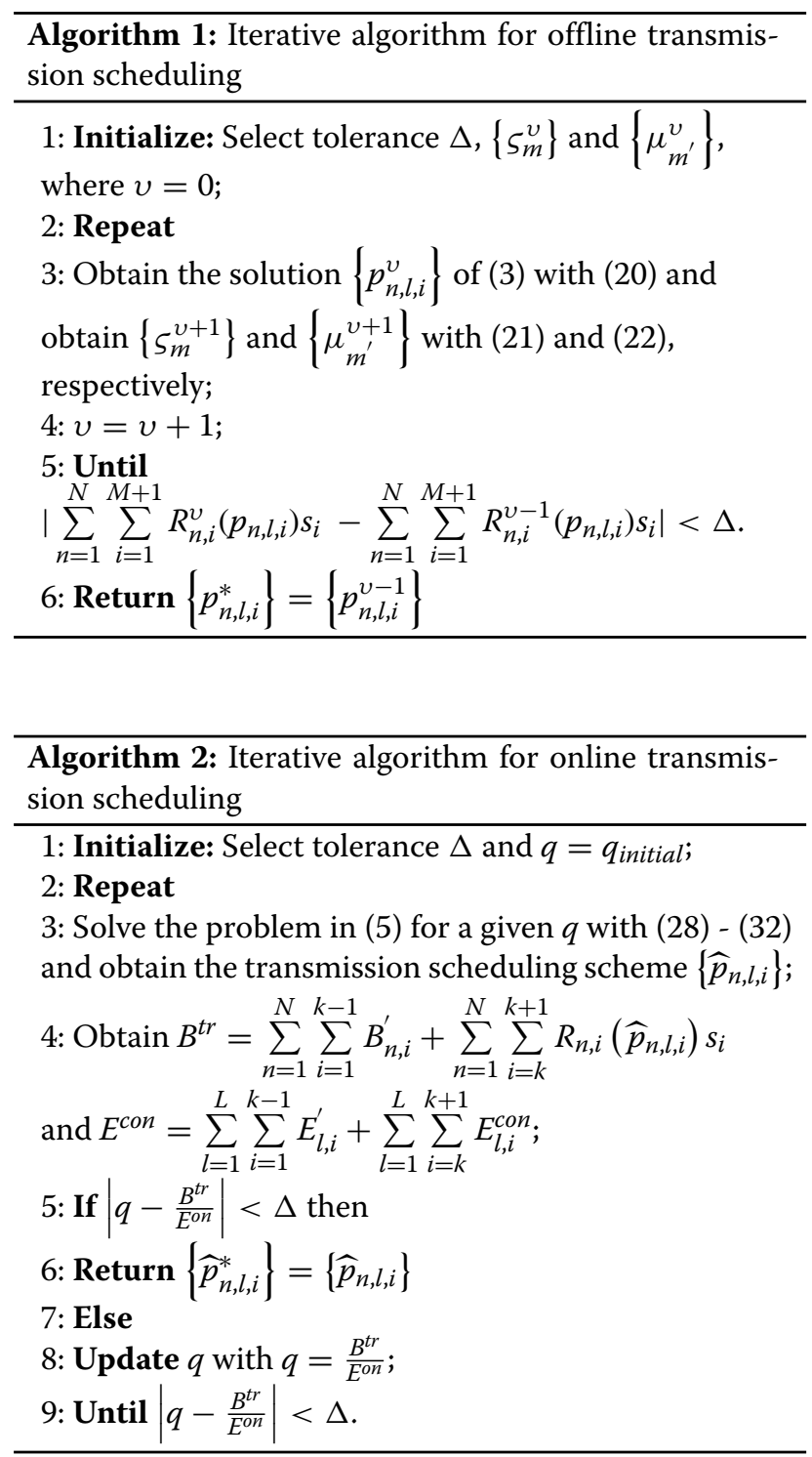

\section{Performance evaluation and analysis}

In this section, the performance of the proposed transmission scheduling algorithms are evaluated in a deterministic energy arrival sequence setting and compared with single RRU transmission (SRT) algorithms with a priori knowledge and limited pre-knowledge about CSI. Besides, we also compare the proposed online algorithm with the Markov decision process (MDP)-based algorithm [35]. It is assumed that energy arrives at time $t=[1,2,3, \ldots, 9] \mathrm{s}$ with different amount for each RRU and the total transmission time $T=10 \mathrm{~s}$. The simulation parameters obtained from $[16,21]$ are listed in Table 1.

We first show the influence that the non-overflow and the causality constraints impose on transmission scheduling. The energy consumption line versus transmission time for the proposed algorithms are given in Figure 6. 


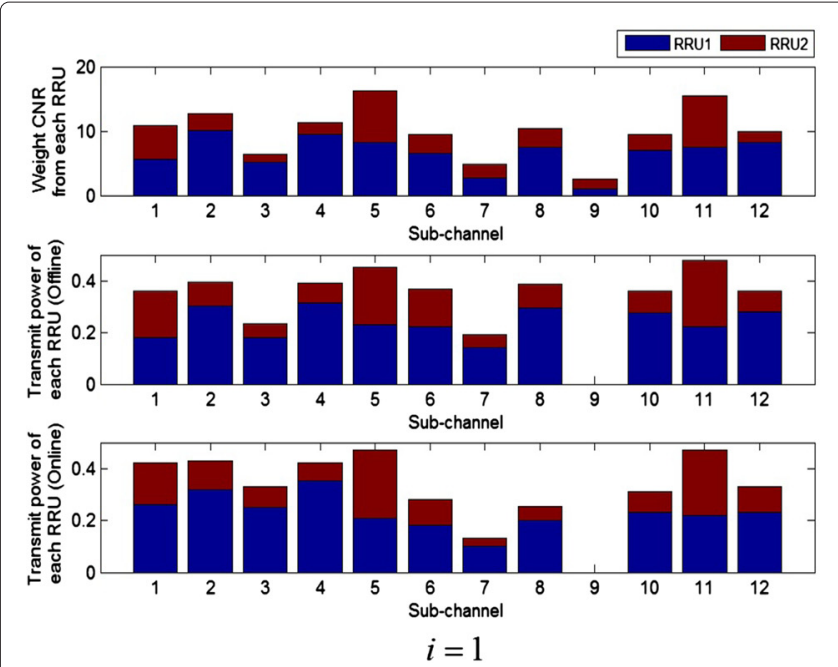

Figure 9 Transmit power allocation versus weighted CNR.

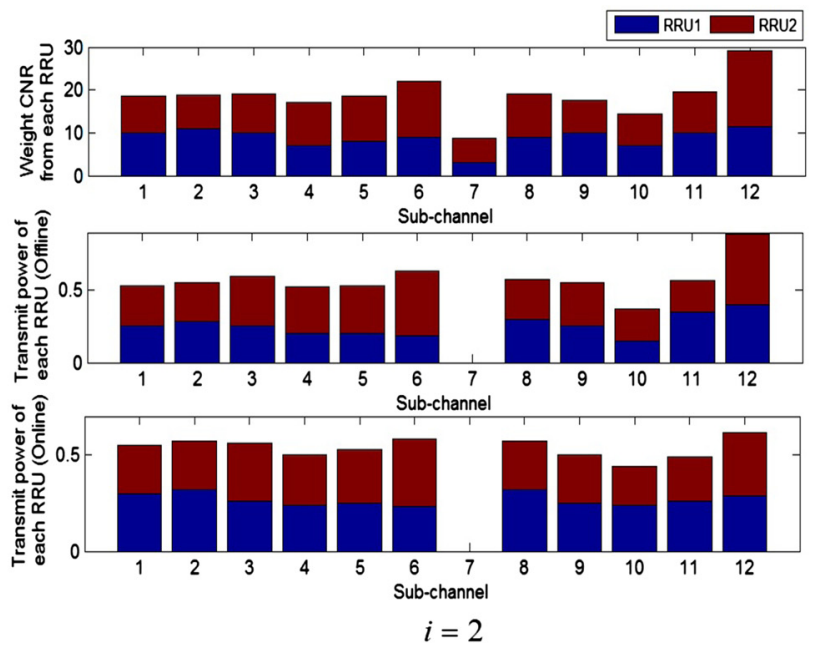

The upper curve in Figure 6 represents the total energy arrived for each RRU, and the lower curve is obtained by subtracting the battery capacity $E_{C}$ from the upper curve. The non-overflow and the causality constraints in (3) and (5) impose the energy consumption line to remain above the lower curve as well as below the upper curve. At the $i$ th time interval, the maximum energy consumption is limited by the total energy arrived before time instant $t_{i}$. Besides, it is also limited by the channel state, e.g., the slope of the energy consumption curve of the proposed offline algorithm for RRU 2 in $[1,2)$ s is larger than in $[0,1) s$, implying that the relatively bad channel state during $[0,1)$ s makes more energy reserve for future use when the channel state is better. What is more, it can be observed that both the proposed offline algorithm and the proposed online algorithm strictly satisfy the causality and non-overflow constraints for each RRU.

We define the energy utilization efficiency as the ratio of the energy consumed for transmission to the currently available energy. As shown in Figure 7, all algorithms achieve high energy utilization efficiency with the minimum of $80 \%$ and reach $100 \%$ at the end of the transmission period. This reason is that, to maximize the total number of transmitted bits, all algorithms are trying to make the best of the available energy. It can also be obtained that the proposed algorithms achieve higher energy utilization efficiency than the algorithms under SRT. It is shown that the maximum difference value between the proposed offline algorithm and the algorithm under SRT with a priori knowledge about CSI is $4.3 \%$ and the maximum

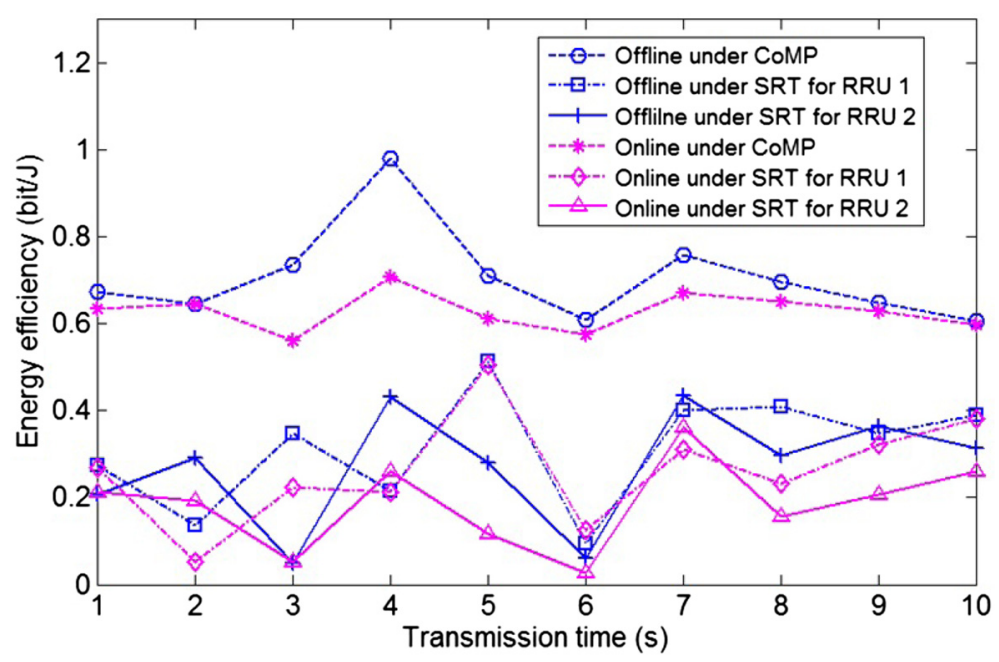

Figure 10 Energy efficiency versus transmission time. 


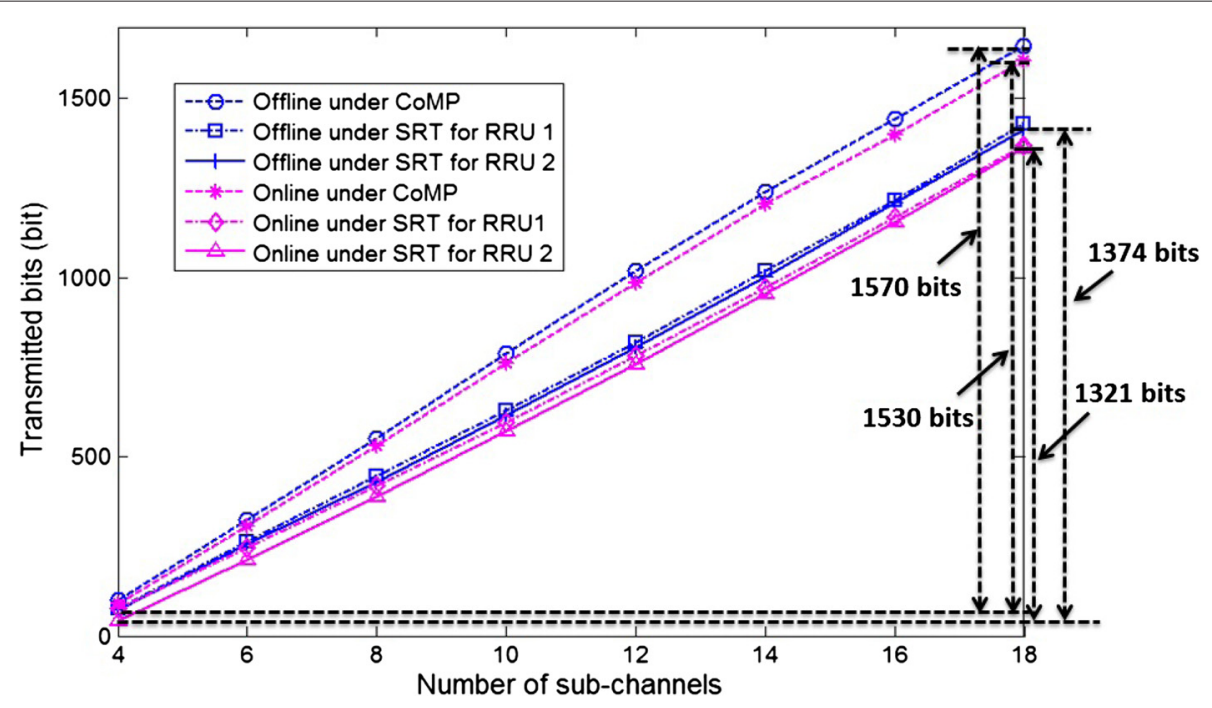

Figure 11 Transmitted bits versus number of sub-channels.

difference value between the proposed online algorithm and the algorithm under SRT with limited pre-knowledge about CSI is $5.8 \%$.

Figure 8 depicts the number of bits transmitted during $[0, T)$ versus transmission time. It can be observed that the proposed online algorithm has a performance close to that of the proposed offline algorithm. The gap between them is no more than 69 bits which brought by the priori knowledge about CSI in the assumption of the offline algorithm. It can be also observed that, the gap between the proposed offline algorithm and the proposed online algorithm is more stable than the gap among the algorithms under SRT. This phenomenon is due to that the SRTs are more easily influenced by channel conditions. In other words, the diversity of channel conditions in CoMP transmission can be explored to enhance the system performance. This analysis can also be verified by observing the slope variation trend of the transmitted bits curve which indicates the relationship between the data rate and the channel state at each time interval.

Figure 9 demonstrates a snapshot of the power distribution across sub-channels and RRUs for the proposed algorithms at the time intervals $i=1$ and $i=2$. It can be observed that the larger the sum of the weighted

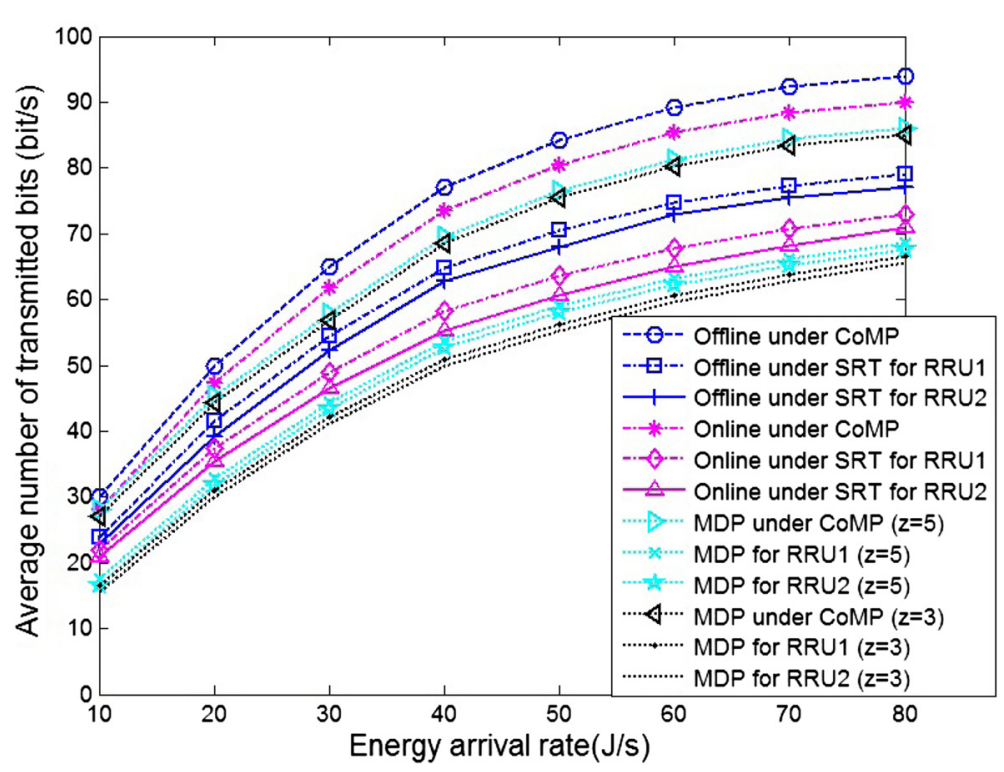

Figure 12 Average number of transmitted bits versus energy arrival rate. 


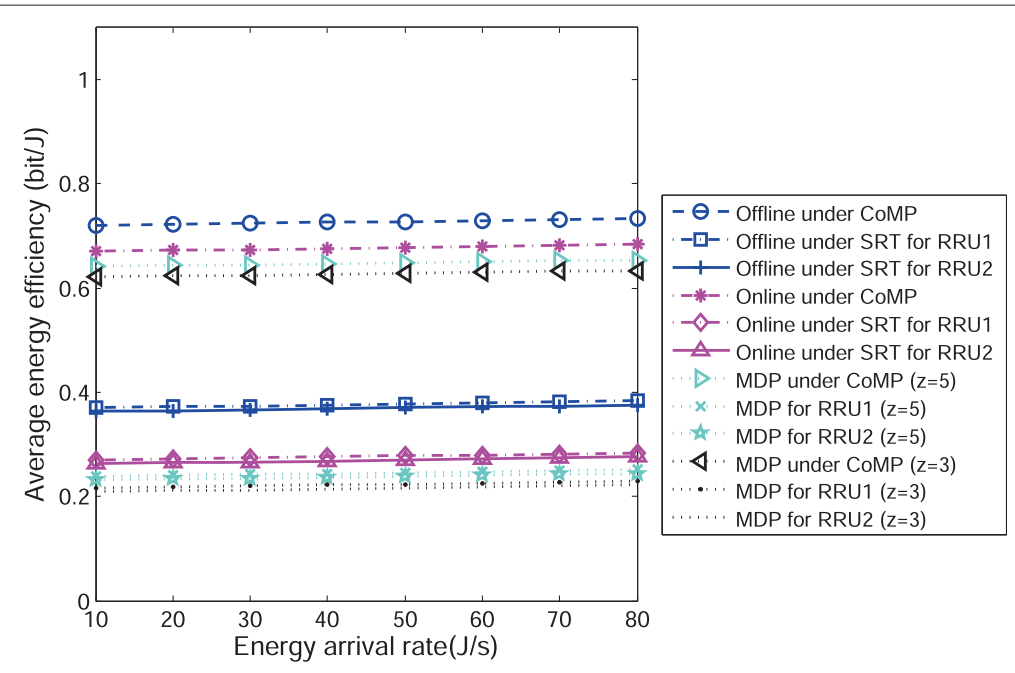

Figure 13 Average energy efficiency versus energy arrival rate.

CNRs from both of the RRUs is, the more the sum of the transmit power on this sub-channel is. Note that, for each sub-channel, the larger the weighted CNR from RRU is, the more the transmit power from the same RRU is. This is due to the fact that at the $i$ th time interval, for sub-channel $n$, the transmit power from each RRU is proportional to the ratio of weighted CNR from the same RRU, as described in (15).

Figure 10 shows the energy efficiency at each time interval versus transmission time and Figure 11 shows the number of transmitted bits versus number of subchannels. It can be observed that CoMP transmission not only achieves higher energy efficiency but transmits more bits than SRTs which prove again that the CoMP transmission is helped for achieving a better system performance. Besides, it can be observed that the larger the number of sub-channels is, the more the bits are transmitted. For instance, compared with four sub-channels, more bits are transmitted under the condition of 18 sub-channels, with increments of 1,570 bits and 1,530 bits for the proposed offline algorithm and the proposed online algorithm, respectively.

The statistical performances of the proposed algorithms are illustrated in the following two figures and compared with the MDP-based algorithm with different number of battery levels, denoted as $z$, which determines the size of the action space. Figure 12 shows the average number of transmitted bits, which are calculated as $\sum_{n=1}^{N} \sum_{i=1}^{M+1} R_{n, i}\left(p_{n, l, i}^{*}\right) s_{i} / T$ and $\sum_{n=1}^{N} \sum_{i=1}^{M+1} B_{n, i}^{\prime}\left(\widehat{p}_{n, l, i}^{*}\right) / T$ for the proposed offline algorithm and the online algorithm, respectively. It can be observed that the performance of the proposed online algorithm approaches the proposed offline algorithm and especially in the low energy arrival rate region. This is because in this case, the transmission scheduling is limited to the amount of available energy which can be used for transmission, and knowledge about the future CSI in the offline algorithm is less valuable for a transmission scheduling purpose. While in the high energy arrival rate region, the high energy arrival rate converts the battery into a continuous energy source. As a result, the influence of knowledge about the future CSI in the offline algorithm becomes significant.

The average energy efficiency of the proposed offline algorithm and the online algorithm is obtained by $s_{i} \sum_{n=1}^{N} \sum_{i=1}^{M+1} R_{n, i}\left(p_{n, l, i}^{*}\right) / \sum_{l=1}^{L} \sum_{i=1}^{M+1} E_{l, i}^{c o n}\left(p_{n, l, i}^{*}\right)$ and $\sum_{n=1}^{N} \sum_{i=1}^{M+1}$ $B_{n, i}^{\prime}\left(\widehat{p}_{n, l, i}^{*}\right) / \sum_{l=1}^{L} \sum_{i=1}^{M+1} E_{l, i}^{\prime}\left(\widehat{p}_{n, l, i}^{*}\right)$, respectively, which are provided in Figure 13 and compared with the MDPbased algorithm with varied energy arrival rates. It can be observed that the proposed offline/online algorithm achieves similar average energy efficiency whether in the low energy arrival rate region or in the high energy arrival rate region. This is because both the offline and the online

Table 2 Comparisons of average energy efficiency, average data rate, and reduced $\mathrm{CO}_{2}$ emissions of different algorithms

\begin{tabular}{llll}
\hline & $\mathbf{I 1}^{\mathbf{a}}$ & $\mathbf{I 2}^{\mathbf{b}}$ & $\mathbf{I 3}^{\mathbf{c}}$ \\
\hline Offline under CoMP & 0.71 & 109.06 & 3.36 \\
Online under CoMP & 0.62 & 102.25 & 3.14 \\
SRT with a priori knowledge about CSI & 0.29 & 73.17 & 1.17 \\
SRT with limited pre-knowledge about CSI & 0.22 & 61.95 & - \\
\hline
\end{tabular}

al1 stands for average energy efficiency, measured in bit/J.

${ }^{\mathrm{b}} / 2$ stands for average data rate, measured in bit/s.

${ }^{\mathrm{C}} \mathrm{3}$ stands for reduced $\mathrm{CO}_{2}$ emission, measured in $\times 10^{-4} \mathrm{~g} / \mathrm{bit}$. 
algorithms are trying to make the best of the available energy obtained during the transmission. What is more, it can be seen from Figures 12 and 13 that the performance of the MDP-based algorithm can be improved as the number of battery level, $z$, increases. In addition, it can also be obtained that the performance of the proposed online algorithm outperforms the MDP-based algorithm, due to the fact that the proposed online algorithm is immune to the number of battery levels.

Table 2 lists the comparisons of the average energy efficiency, average data rate, and $\mathrm{CO}_{2}$ emissions of the proposed algorithms. With the latest estimates from the International Energy Agency, each kWh of electricity coming from the electric grid produces roughly $386 \mathrm{~g}$ of $\mathrm{CO}_{2}$ [32]. Compared with the algorithm under SRT (averaged over RRU1 and RRU2) with limited pre-knowledge about CSI, for every bit transmitted, the reduced $\mathrm{CO}_{2}$ emissions are $3.36 \times 10^{-4} \mathrm{~g}, 3.14 \times 10^{-4} \mathrm{~g}$, and $1.17 \times 10^{-4} \mathrm{~g}$ for the proposed offline algorithm, the proposed online algorithm, and the algorithm under SRT with a priori knowledge about CSI, respectively.

\section{Conclusions}

In this paper, we propose a new REH enabled transmission framework powered by hybrid energy sources including on-grid energy source and off-grid renewable energy. Based on the framework, the joint transmission and harvested energy scheduling algorithms are investigated for the hybrid energy powered cellular transmission system under CoMP transmission. Firstly, we formulate an optimal offline transmission scheduling problem with a priori knowledge about CSI. Considering practical constraint of limited pre-knowledge about CSI, we further transform the offline problem into an energy-aware energy-efficient transmission problem. By extending convex optimization method and non-linear fractional programming to the offline problem and the online problem, respectively, we design two joint transmission and energy scheduling algorithms. Moreover, we prove that at each transmission time interval during the finite transmission period, the transmit power of each RRU is proportional to the weighted CNR of each sub-channel. Numerical results show that the performance of the proposed online algorithm is close to that of the proposed offline algorithm and outperforms the MDP-based algorithm. Besides, the proposed REH enabled transmission framework not only reduces the $\mathrm{CO}_{2}$ emissions but also improves the energy utilization efficiency of renewable energy.

So far, the transmission scheduling problems have been considered with the assumption that all data packets have arrived at RRUs before transmissions begin. Interesting topics for future work include investigating joint transmission and harvested energy scheduling algorithm for random data packets arrival scenario where data packets arrive at arbitrary time instants during the course of transmission and each packet contains an individual transmission constraint. As RRU cannot transmit the data that has not arrived yet, the transmission algorithm is not only subject to individual transmission delay constraint but also to transmission causality constraint. The design of the transmission scheduling algorithm becomes even more challenging when taking into account the random characteristics of data packet arrival time and transmission delay requirement.

\section{Competing interests}

The authors declare that they have no competing interests.

\section{Acknowledgements}

This work was supported by the National Nature Science Foundation of China (No. 61302108), US National Science Foundation $(1145596,0830493)$ and National Key Technology R\&D Program (2012BAH06B02).

\section{Author details}

${ }^{1}$ State Key Laboratory of Information Security, Institute of Information Engineering, Chinese Academy of Sciences, Beijing 100093, China. ${ }^{2}$ Cisco Systems, Inc., Beijing 100022, China. ${ }^{3}$ Department of CEEN, University of Nebraska - Lincoln, Lincoln, NE, 68182, USA.

Received: 20 September 2014 Accepted: 2 March 2015

Published online: 01 April 2015

\section{References}

1. H Klessig, A Fehske, GP Fettweis, in Sarnoff Symposium. Energy efficiency gains in interference-limited heterogeneous cellular mobile radio networks with random micro site deployment (IEEE, Piscataway, NJ, 2011), pp. 1-6

2. L Wang, C Singh, Multicriteria design of hybrid power generation systems based on a modified particle swarm optimization algorithm. Energy Conversion IEEE Trans. 24(1), 163-172 (2009)

3. Z Hasan, H Boostanimehr, VK Bhargava, Green cellular networks: A survey, some research issues and challenges. Commun. Surv. Tutor. IEEE. 13(4), 524-540 (2011)

4. 3GPP TR 32.826 V10.0.0, Technical Specification Group Services and System Aspects; Telecommunication Management; Study on Energy Savings Management (ESM) (Release 10), (2010). Available: http://www.3gpp.org/ $\mathrm{ftp} /$ specs/archive/32_series/32.826/

5. 3GPP TS 36.927, Evolved Universal Terrestrial Radio Access (E-UTRA); Potential Solutions for Energy Saving for E-UTRAN (Release 11), (2012). Available: http://www.3gpp.org/DynaReport/36927.htm

6. Y-H Nam, L Liu, JC Zhang, Cooperative Communications for LTE-advanced Relay and CoMP. Int. J. Commun. Syst. 27(10), 1616-1625 (2014)

7. 3GPP TR36.814 V2.0.1, Evolved Universal Terrestrial Radio Access (E-UTRA); Further Advancements for E-UTRA Physical Layer Aspects, (2010). Available: http://www.3gpp.org/DynaReport/32814.htm

8. Ng DWK, Lo ES, R Schober, in Wireless Communications and Networking Conference (WCNC), 2013 IEEE. Energy-efficient resource allocation in multiuser OFDM systems with wireless information and power transfer (IEEE, 2013), pp. 3823-3828

9. DWK Ng, ES Lo, R Schober, Energy-efficient resource allocation in multi-cell OFDMA systems with limited backhaul capacity. Wireless Commun. IEEE Trans. 11(10), 3618-3631 (2012)

10. C Isheden, Z Chong, E Jorswieck, G Fettweis, Framework for link-level energy efficiency optimization with informed transmitter. Wireless Commun. IEEE Trans. 11(8), 2946-2957 (2012)

11. KTK Cheung, S Yang, L Hanzo, Achieving maximum energy efficiency in multi relay OFDMA cellular networks: A fractional programming approach. Commun. IEEE Trans. 61(7), 2746-2757 (2013)

12. $S$ He, Y Huang, $S$ Jin, L Yang, Coordinated beamforming for energy efficient transmission in multicell multiuser systems. Commun. IEEE Trans. 61(12), 4961-4971 (2013) 
13. Z Xu, C Yang, GY Li, Y Liu, S Xu, Energy-efficient comp precoding in heterogeneous networks. Signal Process. IEEE Trans. 62(4), 1005-1017 (2014)

14. KTutuncuoglu, A Yener, Optimum transmission policies for battery limited energy harvesting nodes. Wireless Commun. IEEE Trans. 11(3), 1180-1189 (2012)

15. O Ozel, K Tutuncuoglu, J Yang, S Ulukus, A Yener, Transmission with energy harvesting nodes in fading wireless channels: Optimal policies. Selected Areas Commun. IEEE J. 29(8), 1732-1743 (2011)

16. O Ozel, J Yang, S Ulukus, Optimal broadcast scheduling for an energy harvesting rechargeable transmitter with a finite capacity battery. Wireless Commun. IEEE Trans. 11(6), 2193-2203 (2012)

17. J Yang, O Ozel, S Ulukus, Broadcasting with an energy harvesting rechargeable transmitter. Wireless Commun. IEEE Trans. 11(2), 571-583 (2012)

18. T Han, N Ansari, Ice: Intelligent cell breathing to optimize the utilization of green energy. Commun. Lett. IEEE. 16(6), 866-869 (2012)

19. THan, X Huang, N Ansari, in Communications (ICC). Energy agile packet scheduling to leverage green energy for next generation cellular networks (IEEE, Piscataway, NJ, 2013), pp. 3650-3654

20. J Gong, S Zhou, Z Niu, JS Thompson, in Vehicular Technology Conference (VTC Spring). Energy-aware resource allocation for energy harvesting wireless communication systems (IEEE, Piscataway, NJ, 2013), pp. 1-5

21. DWK Ng, ES Lo, Schober R, Energy-efficient resource allocation in OFDMA systems with hybrid energy harvesting base station. Wireless Commun. IEEE Trans. 12(7), 3412-3427 (2013)

22. C Huang, R Zhang, S Cui, Throughput maximization for the gaussian relay channel with energy harvesting constraints. Selected Areas Commun. IEEE J. 31(8), 1469-1479 (2013)

23. D Gunduz, B Devillers, in Computational Advances in Multi-Sensor Adaptive Processing (CAMSAP). Two-hop communication with energy harvesting (IEEE, Piscataway, NJ, 2011), pp. 201-204

24. J Xu, Y Guo, R Zhang, in Global Communications Conference (GLOBECOM). Comp meets energy harvesting: a new communication and energy cooperation paradigm (IEEE, Piscataway, NJ, 2013), pp. 2508-2513

25. C Huang, R Zhang, S Cui, Optimal power allocation for outage probability minimization in fading channels with energy harvesting constraints. Wireless Commun. IEEE Trans. 13(2), 1074-1087 (2014)

26. S Jafarzadeh, MS Fadali, CY Evrenosoglu, Solar power prediction using interval type-2 tsk modeling. Sustainable Energy IEEE Trans. 4(2), 333-339 (2013)

27. H-T Yang, C-M Huang, Y-C Huang, Y-S Pai, A weather-based hybrid method for 1-day ahead hourly forecasting of pv power output. Sustainable Energy IEEE Trans. 5(3), 917-926 (2014)

28. H Zhang, C Jiang, NC Beaulieu, X Chu, Resource Allocation for Cognitive Small Cell Networks: A Cooperative Bargaining Game Theoretic Approach. Wireless Commun. IEEE Trans. (2015). doi:10.1109/TWC.2015.2407355

29. D Schafhuber, G Matz, Mmse and adaptive prediction of time-varying channels for OFDM systems. Wireless Commun. IEEE Trans. 4(2), 593-602 (2005)

30. I Wong, BL Evans, Sinusoidal modeling and adaptive channel prediction in mobile OFDM systems. Signal Process. IEEE Trans. 56(4), 1601-1615 (2008)

31. S Boyd, L Vandenberghe, Convex Optimization. (Cambridge University Press, UK, 2009)

32. G Piro, M Miozzo, G Forte, N Baldo, LA Grieco, G Boggia, P Dini, Hetnets powered by renewable energy sources: sustainable next-generation cellular networks. Internet Comput. IEEE. 17(1), 32-39 (2013)

33. W Dinkelbach, On nonlinear fractional programming. Manage. Sci. 13(7), 492-498 (1967)

34. DWK Ng, ES Lo, R Schober, Energy-efficient resource allocation for secure OFDMA systems. Vehicular Technol. IEEE Trans. 61(6), 2572-2585 (2012)

35. A Minasian, S Shahbazpanahi, RS Adve, Energy harvesting cooperative communication systems. Wireless Commun. IEEE Trans. 13(11), 6118-6131 (2014)

\section{Submit your manuscript to a SpringerOpen ${ }^{\mathcal{O}}$ journal and benefit from:}

- Convenient online submission

- Rigorous peer review

- Immediate publication on acceptance

- Open access: articles freely available online

- High visibility within the field

- Retaining the copyright to your article

Submit your next manuscript at $\boldsymbol{\wedge}$ springeropen.com 